$1-1-1998$

\title{
Theoretical foundation of the optical current-controlled oscillator and its applications for subcarrier optical communications
}

\author{
Guifang Li \\ University of Central Florida \\ Feng Tian
}

Find similar works at: https://stars.library.ucf.edu/facultybib1990 University of Central Florida Libraries http://library.ucf.edu

This Article is brought to you for free and open access by the Faculty Bibliography at STARS. It has been accepted for inclusion in Faculty Bibliography 1990s by an authorized administrator of STARS. For more information, please contact STARS@ucf.edu.

\section{Recommended Citation}

$\mathrm{Li}$, Guifang and Tian, Feng, "Theoretical foundation of the optical current-controlled oscillator and its applications for subcarrier optical communications" (1998). Faculty Bibliography 1990s. 2324. https://stars.library.ucf.edu/facultybib1990/2324

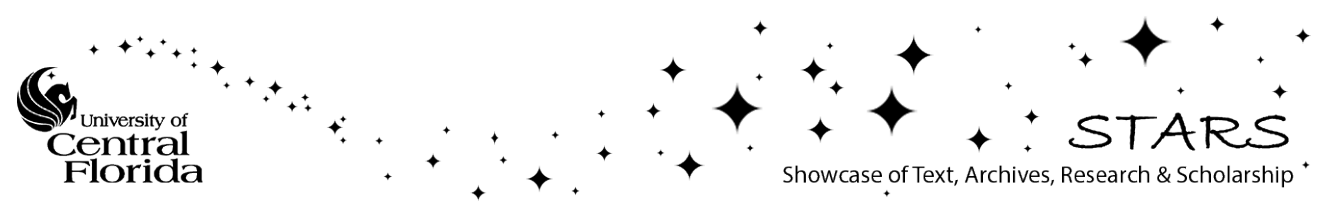




\section{Theoretical foundation of the optical current- controlled oscillator and its applications for subcarrier optical communications}

Cite as: Journal of Applied Physics 83, 6140 (1998); https://doi.org/10.1063/1.367483

Submitted: 16 July 1997 . Accepted: 04 March 1998. Published Online: 13 October 1998

Guifang $\mathrm{Li}$, and Feng Tian

Lock-in Amplifiers

... and more, from DC to $600 \mathrm{MHz}$ 


\title{
Theoretical foundation of the optical current-controlled oscillator and its applications for subcarrier optical communications
}

\author{
Guifang $\mathrm{Li}^{\mathrm{a})}$ \\ Center for Research and Education in Optics and Lasers, University of Central Florida, Orlando, \\ Florida 32816-2700 \\ Feng Tian \\ Newbridge Networks Corporation, 600 March Road, P.O. Box 13600, Kanata, Ontario, K2K 2E6, Canada
}

(Received 16 July 1997; accepted for publication 4 March 1998)

\begin{abstract}
The optical current-controlled oscillator (CCO) is an all-optical device capable of producing frequency-tunable intensity oscillations (subcarriers) on an optical carrier without any active radio frequency/microwave components. From a dynamic point of view, the CCO is a nonlinear optical system supporting the Hopf bifurcation and operating in the periodic orbit regime. This article presents the theoretical foundation for the optical $\mathrm{CCO}$ and its applications in optical communications involving subcarriers. Using one embodiment of the optical $\mathrm{CCO}$ employing a two-section Fabry-Pérot laser diode, we present the static and modulation characteristics of the periodic-orbit subcarrier of the optical CCO. The performance of CCO-based all-optical subcarrier optical communication system is estimated using representative device parameters. The relationship between the capacity of CCO-based communication networks and the frequency-tuning range of the CCO is also established. (C) 1998 American Institute of Physics. [S0021-8979(98)05711-9]
\end{abstract}

\section{INTRODUCTION}

Radio frequency (rf) and microwave/millimeter-wave (MMW) modulated optical signals frequently appear in optical communications. ${ }^{1-3}$ For example, subcarrier multiplexing (SCM)/multiaccess (SCMA) optical networks are created by using the frequency of the rf/MMW subcarrier of each node as its address. ${ }^{4,5}$ In general, the rf/MMW subcarrier is an auxiliary or supporting component, initially in the electronic format. The information intended for it first modulates the frequency or the amplitude of the subcarrier; the information-bearing subcarrier then modulates the intensity of the optical carrier. Up until recently, rf/MMW subcarriers have been generated using either electronic devices or optical heterodyne detection techniques. In particular, in previous demonstrations of SCM/SCMA optical networks, rf/MMW subcarriers have been generated electronically using devices such as voltage controlled oscillators (VCOs), comb generators and filter banks. ${ }^{6}$ Microwave/millimeter-wave modulated optical signals also appear in antenna remoting, ${ }^{7}$ photonic packet switching with subcarrier addressing, ${ }^{8,9}$ subcarrier wavelength routing, ${ }^{10}$ and broadcasting networks such as in the distribution of analog television signals over passive optical networks. ${ }^{11-14}$

Recently, some research efforts have been initiated for realizing "all-optical" SCM/SCMA networks in which optical technology (rather than electronics) plays the major role in network functionality including subcarrier generation. ${ }^{15-21}$ In a conventional sense, "all-optical" and "SCM" seem to be incompatible with each other because of the rf/MMW subcarriers involved. The key device used in Refs. 15-21 for optical subcarrier generation is the self-sustained pulsating

\footnotetext{
a)Electronic mail: li@creol.ucf.edu
}

(SSP) laser diode (LD). Self-pulsation is not an isolated anomaly in the LD dynamics but a robust nonlinear dynamic phenomenon - the formation of periodic orbits. As will be shown in this article, the SSP LD is a member of a class of optoelectronic devices we have termed the optical currentcontrolled oscillator (CCO) which is ideally suited for optical communications involving subcarriers. An optical CCO is an all-optical device capable of generating tunable rf/ MMW subcarriers on an optical carrier without any active rf/MMW components. The optical CCO is a relatively new concept and technology. The purpose of this article is to (a) establish the theoretical foundation for the optical CCO from a device physics and nonlinear dynamics point of view, and (b) illustrate its applications and performance in optical communications involving rf/MMW subcarriers. It is organized as follows: The principle of operation of the optical CCO is presented using a nonlinear-dynamic approach in Sec. II. One realization of the optical CCO using a two-section Fabry-Pérot (FP) laser diode is described in Sec. III. Sections IV and V contain the static and modulation characteristics of the periodic-orbit subcarrier of the optical CCO, respectively. The performance of frequency-modulated subcarrier optical communication systems is estimated using representative device parameters in Sec. VI. Section VII considers network-related issues using the optical CCO. Finally, concluding remarks and discussion are given in Sec. VIII.

\section{PRINCIPLE OF OPERATION OF THE OPTICAL CCO}

To understand the principle of operation of optical CCOs, let us consider dynamic behaviors of nonlinear systems in general. Figure 1 depicts a typical sequence of dynamic behaviors of a nonlinear system as one parameter (control parameter) of the system is changed. Three charac- 


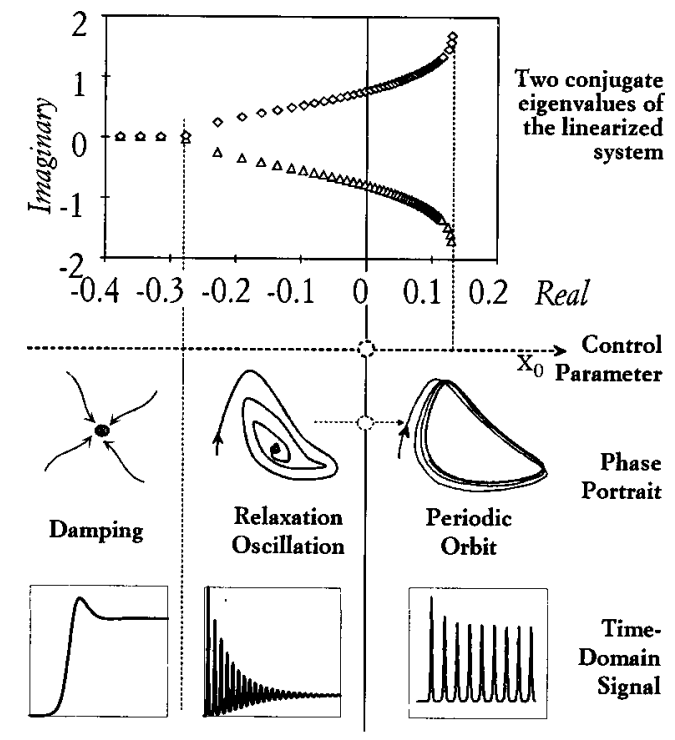

FIG. 1. A typical sequence of dynamic behaviors of a nonlinear system as one parameter of the system is changed.

teristic regions are shown as indicated by the nature of the two conjugate eigenvalues of the linearized system, about the equilibrium of the original nonlinear system, ${ }^{22}$ as well as the corresponding phase portraits and the time-domain signals. (There are as many eigenvalues as there are degrees of freedom of the system. All other eigenvalues are assumed to be real and negative and thus do not affect the stability of the linearized system.) For small values of the control parameter, the eigenvalues are real and negative and the nonlinear system is stable. It responds to a step-function input with a damping transient toward the equilibrium point (fixed point). As the control parameter is increased, the two eigenvalues become complex (conjugates of each other). As long as the real part of the eigenvalues remains negative, the system is still linearly stable and undergoes relaxation oscillations toward the fixed point. As the control parameter is further increased, the real part of the two conjugate eigenvalues becomes positive. The system then becomes linearly unstable; however, it is nonlinearly stable and leads to the formation of periodic orbits. The bifurcation from relaxation oscillations to periodic orbits is called a Hopf bifurcation. ${ }^{23}$ The frequency and amplitude of the periodic orbits are, in general, functions of the control parameter of the system. As a result, the frequency and amplitude of the periodic orbits can be modulated by changing the control parameter for information encoding for communication purposes. In optical CCOs involving LDs as nonlinear elements, the periodic orbit corresponds to the rf/MMW intensity oscillations and the control parameter is the bias current. The dc bias establishes the frequency of rf/MMW intensity oscillations (subcarriers) on the optical carrier without using any active rf/MMW components. The information in its electric form superimposed on the dc bias then modulates the frequency and the amplitude of the MMW subcarrier. In what follows, we shall illustrate the principle of operation, the characteristics, and applications of optical CCOs using the two-section FP LD.

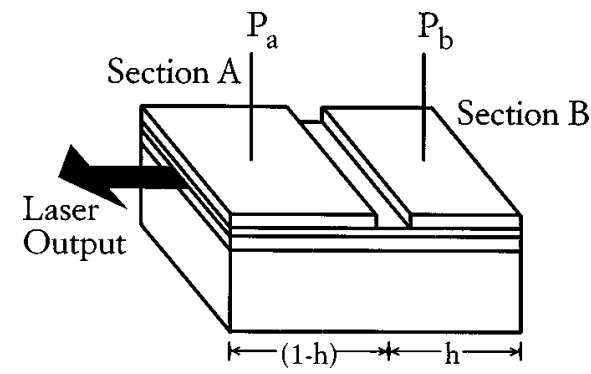

FIG. 2. Schematic of an optical CCO composed of an amplifying section A and an absorbing section B within a common Fabry-Pérot cavity. $P_{a}$ and $P_{b}$ are the pumping rates and $h$ is the volume fraction of section B.

\section{REALIZATION OF THE OPTICAL CCO- TWO-SECTION FP LD: AN EXAMPLE}

Since the dynamics from damping to relaxation oscillations to periodic orbits are ubiquitous in nonlinear systems, it is expected that many optoelectronic devices involving laser diodes will support the formation of periodic orbits. In other words, there are potentially many device arrangements involving laser diodes that will function as optical CCOs. The two-section laser diode shown in Fig. 2 is one realization of an optical CCO. The two-section FP LD is believed to be a model for SSP LDs, ${ }^{23-27}$ even though we will show in Sec. IV that the frequency dependence of two-section FP LDs differs from that of SSP LDs measured experimentally.

As shown in Fig. 2, the two-section FP LD is formed by cascading an amplifying section $\mathrm{A}$ and an absorbing section B in a common Fabry-Pérot resonator. We denote $n_{a}$ and $n_{b}$ the average carrier densities in sections $\mathrm{A}$ and $\mathrm{B}$, respectively. Assuming that the laser operates in single mode, the rate equations for the carrier densities and average photon density $N$ in the cavity are given as follows:

$$
\begin{aligned}
& \frac{d n_{a}}{d t}=-\frac{n_{a}}{\tau_{a}}-G_{a}\left(n_{a}\right) N+P_{a}, \\
& \frac{d n_{b}}{d t}=-\frac{n_{b}}{\tau_{b}}-G_{b}\left(n_{b}\right) N+P_{b}, \\
& \frac{d N}{d t}=\left[(1-h) G_{a}\left(n_{a}\right)+h G_{b}\left(n_{b}\right)-\Gamma\right] N,
\end{aligned}
$$

where $\tau_{s}$ and $P_{s}[s=a($ section A) or $b$ (section B)] are the carrier lifetimes and the carrier injection rates in the respective sections, $h$ is the volume fraction of the absorbing section in the cavity, and $\Gamma$ is the cavity optical loss. $G_{a}\left(n_{a}\right)$ and $G_{b}\left(n_{b}\right)$ are gain values related to the carrier density in sections A and B, respectively, and are approximated as linear functions: $G_{s}\left(n_{s}\right)=g_{s}\left(n_{s}-n_{0 s}\right)$, where $g_{s}=g_{s 0} /(1$ $\left.+\epsilon_{N L} N\right)$ is the differential gain $\left[g_{s}=d G_{s}\left(n_{s}\right) / d n_{s}\right]$, and $\epsilon_{N L}$ is the gain compression coefficient, and $n_{0 s}$ is the carrier density at [which $G_{s}\left(n_{s}\right)$ vanishes] transparency.

A standard linear stability analysis can be used to determine the parameter space for the three dynamic behaviors schematically illustrated in Fig. 1 for the two-section FP LD. ${ }^{17}$ The standard procedures for the linear stability analy- 


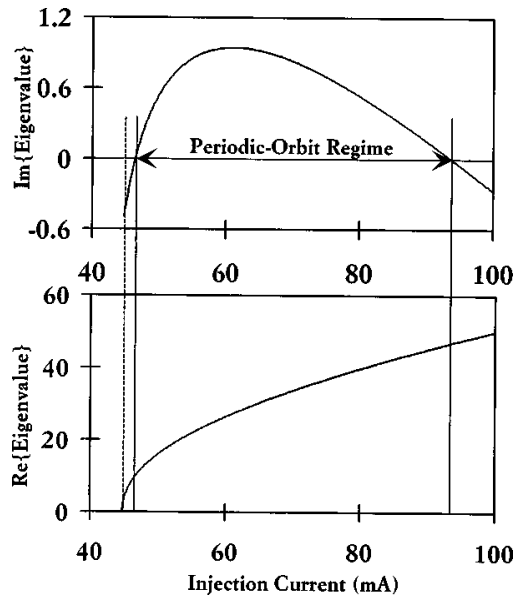

FIG. 3. The real and imaginary parts of one of the three eigenvalues as functions of the injection current in the amplifying section.

sis lead to a linear system - a set of linear ordinary differential equations-governing the perturbation $\epsilon=\left(\delta n_{a}\right.$, $\left.\delta n_{b}, \delta N\right)^{T}$ from the equilibrium solution:

$$
d \epsilon / d t=J \times \epsilon,
$$

where $J$ is called the Jacobian of the original nonlinear system [Eqs. (1)-(3)] about the equilibrium. The nature of the eigenvalues, $\lambda_{j}(j=1,2,3)$, of the Jacobian determines the stability of the equilibrium solution.

As a practical example, we chose the following device parameters consistent with the experimental device used previously: ${ }^{15-21}$ cavity volume $V=250 \times 4 \times 0.2 \mu \mathrm{m}, h$ $=0.025, \tau_{a}=2 \quad \mathrm{~ns}, \quad \tau_{b}=0.1 \mathrm{~ns}, n_{0 a}=1.2 \times 10^{18} \mathrm{~cm}^{-3}$, $n_{0 b}=2.4 \times 10^{18} \mathrm{~cm}^{-3}, \quad \Gamma=6.25 \times 10^{11} \mathrm{~s}^{-1}, \quad \epsilon_{N L}=0.8$ $\times 10^{-18} \mathrm{~cm}^{3}, \quad g_{0 a}=4.5 \times 10^{-7} \mathrm{~cm}^{3} / \mathrm{s}$, and $g_{b 0} / g_{a 0}=0.05$. The injection current for section $B$ is equal to $1.5 \mathrm{~mA}$. There are three eigenvalues for the linearization about the equilibrium of the original nonlinear system. One of them is always real and negative. The other two are complex conjugates of each other. So only one of the complex eigenvalues (both the real and imaginary parts) is presented in Fig. 3 as the injection current in the amplifying section A is varied. Relaxation oscillation starts at an injection current of $43 \mathrm{~mA}$. The system undergoes a Hopf bifurcation from relaxation oscillations to periodic orbits at an injection current of about $46 \mathrm{~mA}$ where the complex eigenvalues cross the real axis. The Hopf bifurcation is structurally stable with respect to nonlinear gain compression. In other words, periodic orbits exist in two-section FP LDs without gain compression. Gain compression is only included here to represent realistic device parameters. It is not required for successful operation of the optical CCO. When the injection current is decreased from high levels, a similar Hopf bifurcation occurs at an injection current of $93 \mathrm{~mA}$. Figure 4 is a representative dynamic time response at an injection current of $82 \mathrm{~mA}$. Within the region of periodic orbits corresponding to injection currents between 46 and $93 \mathrm{~mA}$, the two-section FP LD functions as an optical CCO. As the injection current is changed, the frequency (amplitude) of the periodic orbits is also changed. These characteristics form the basis for the two-section FP LDs, and the optical CCO in general, for carrying informa-

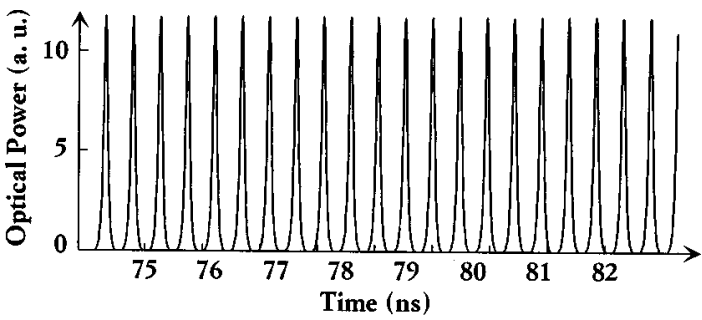

FIG. 4. A sample periodic-orbit dynamic supported by the two-section FP LD. The periodic orbits are used as subcarriers.

tion in subcarrier optical communications as demonstrated below by its static and modulation characteristics.

\section{STATIC CHARACTERISTICS OF THE PERIODIC- ORBIT SUBCARRIER}

The linearized theory is a powerful tool for determining the parameter space for the existence of periodic orbits. It cannot, however, be used to accurately predict the nonlinear characteristics of the periodic orbits. The static characteristics of the periodic-orbit subcarrier presented below are obtained by exact numerical integration of rate equations (1)(3). Unless specified otherwise, the device parameters are chosen to be the same as those used for the eigenvalue analysis of the linearized system in Sec. III.

\section{A. Frequency and amplitude dependence}

The frequency of the periodic orbits increases with the injection current as shown in Fig. 5(a). Since the periodic orbits are not purely sinusoidal (nonharmonic), the frequency plotted in Fig. 5(a) is the fundamental frequency of the periodic orbits. As can be seen, the fundamental frequency increases with the injection current almost quadratically. This behavior is in contrast to the imaginary part of the eigenvalue of the Jacobian of the linearized system where the dependence is parabolic. The measured frequency of selfsustained pulsation in SSP laser diodes shows a parabolic dependence. ${ }^{17,28}$ We have investigated this frequency dependence almost exhaustively in the parameter space where periodic orbits exist and found that, theoretically, this quadratic dependence for the two-section FP LDs is universally true. This discrepancy between the two-section FP model and the SSP LDs seems to suggest that SSP LDs cannot be described completely by the present two-section model as was previously believed to be the case. ${ }^{26}$

As the injection current increases from the lower Hopf bifurcation point, the amplitude of the oscillation increases. It reaches a maximum approximately midway between the two Hopf bifurcation points. This behavior is shown in Fig. 5(b). We observe that the real part of the eigenvalue of the Jacobian also has a similar dependence on the injection current (Fig. 3).

\section{B. Harmonic content}

The wave forms of the nonsinusoidal periodic orbits are determined by their harmonic content. The subcarrier optical communication systems and networks to be described subse- 


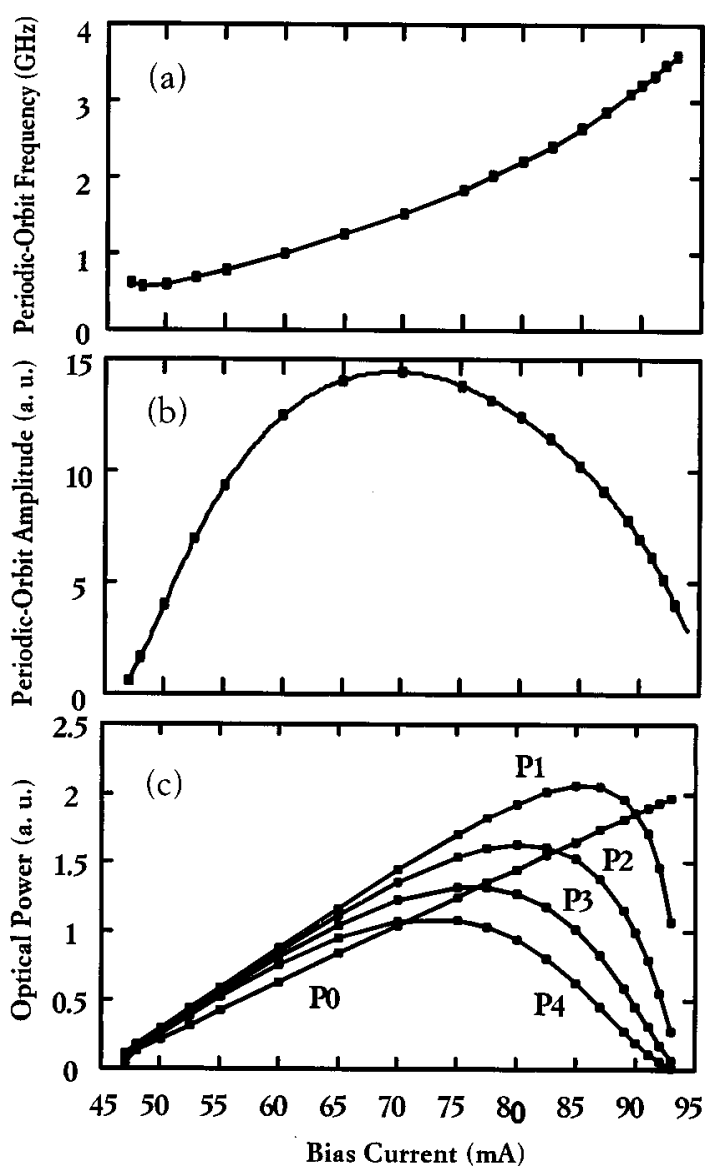

FIG. 5. The frequency (a), amplitude (b), and the harmonic power (c) in the periodic orbits as functions of the injection current in the amplifying section. $P_{0}$ is the dc/average power and $P_{n}$ is the power of the $n$th harmonic.

quently using the optical $\mathrm{CCO}$ will be based on frequency or amplitude modulation of a particular harmonic component so that standard demodulation techniques for amplitude modulation (AM) and frequency modulation (FM) can be used. That is, information will be recovered from only one harmonic in the modulated periodic orbits. It is thus important to determine the harmonic contents of the periodic-orbit subcarriers. Figure 5(c) shows the harmonic contents of the periodic orbits-the Fourier coefficients of the $\mathrm{CCO}$ output power-as functions of the bias current in section A. Because of the phase relationships among the harmonics, a particular harmonic in the periodic orbit attains a maximum amplitude at a different injection current than that at which the periodic-orbit oscillation amplitude itself attains its maximum.

Out of many harmonics in the periodic orbit only one harmonic is used as the subcarrier. The power budget, which is the maximum power loss allowed from the transmitter to the receiver, is related to the power in the "unmodulated" microwave subcarrier. The unmodulated subcarrier power available for each harmonic in the periodic orbit generated by the optical CCO can be significantly higher than the average power. This is apparent from Fig. 5(c). In a wide range of operation of the CCOs, the Fourier coefficients of the harmonics exceed the dc component (average power of the CCO output, $P_{0}$ ). Over a $100 \%$ modulation index occurs
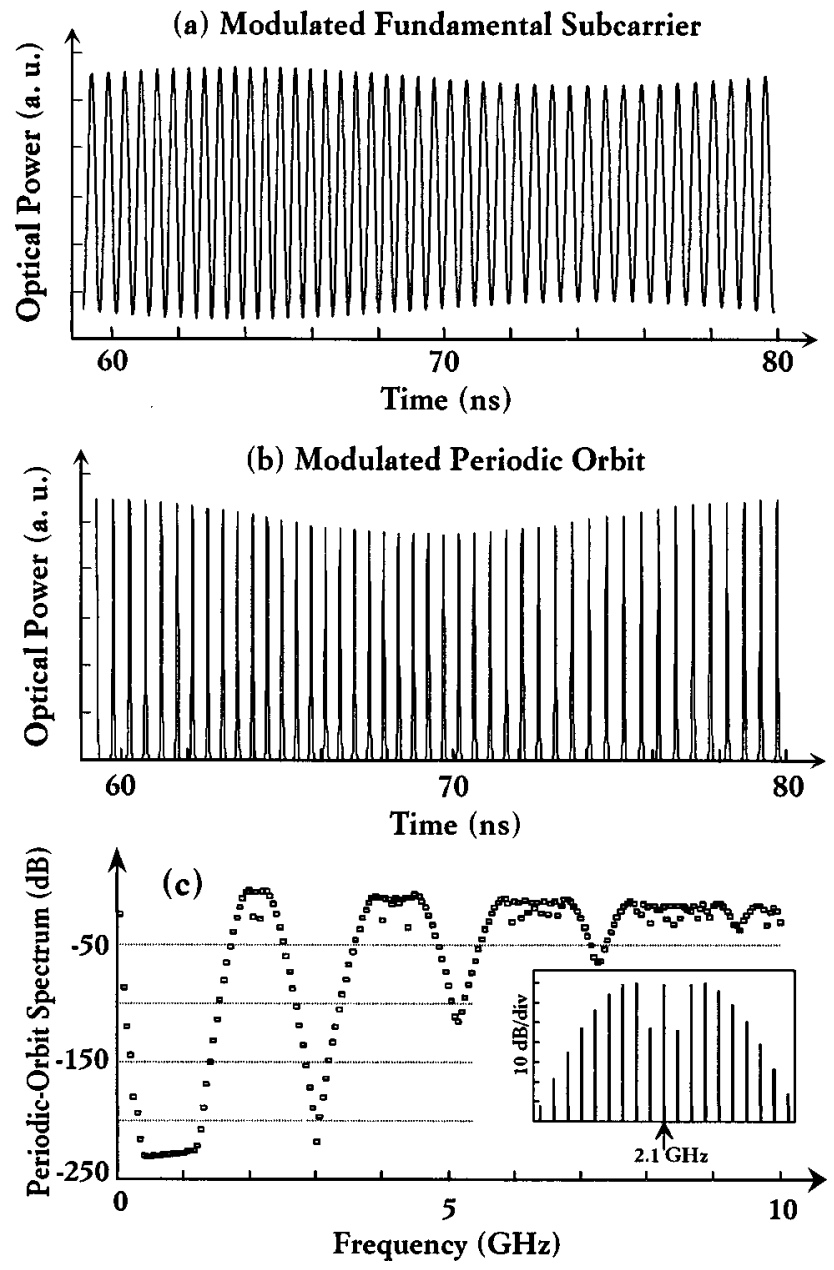

FIG. 6. The modulated fundamental subcarrier (a) and the modulated periodic orbit (b) in the time domain, and the spectrum of the modulated periodic orbit (c). The inset in (c) shows details of the modulated fundamental harmonic near $2.1 \mathrm{GHz}$. The spectral peaks are $50 \mathrm{MHz}$ apart.

naturally in SCM systems based on CCOs. In conventional SCM, the intensity-modulation index is usually below $100 \%,{ }^{29}$ and in practice is limited to $3 \%-4 \%$ per channel to avoid clipping and nonlinearity. ${ }^{30}$ The existence of the harmonics does lead to a restriction on bandwidth efficiency especially for analog transmission as will be shown in Sec. V.

\section{MODULATION CHARACTERISTICS OF THE PERIODIC-ORBIT SUBCARRIER}

When a modulating signal is superimposed on the dc bias of the two-section FP LD, the characteristics of the periodic orbits are modulated. All characteristics of the periodic orbit - the amplitude and frequency of each harmoniccarry information about the modulating signal. This is demonstrated by both the time-domain and frequencydomain representations of the periodic orbit under modulation. In Fig. 6(b), the modulated periodic orbit, the periodic orbit "perturbed" by the modulating signal, is shown in the time domain. The frequency of the free-running periodic orbit subcarrier is $2.1 \mathrm{GHz}$. A sinusoidal signal of frequency $50 \mathrm{MHz}$ and power $-65 \mathrm{~dB} \mathrm{~m}$, corresponding to roughly 6 $\mathrm{mA}$ peak-to-peak deviation, is superimposed on the dc injec- 


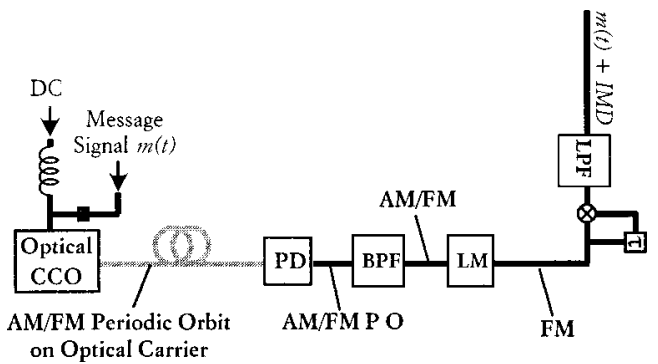

FIG. 7. Schematic of a CCO-based FM subcarrier optical communication system.

tion current of the amplifying region. The peak frequency deviation is about $240 \mathrm{MHz}$. When the modulated periodic orbit is passed through a band pass filter centered at the fundamental frequency of the periodic orbit, the modulated fundamental harmonic (fundamental subcarrier) is obtained and is shown in Fig. 6(a). For this particular optical CCO, the amplitude modulation is almost completely in phase with respect to frequency modulation as instantaneous frequency maxima and instantaneous amplitude minima are attained in the modulated periodic orbit at the same time.

The modulation characteristics of the harmonics are easily seen from the rf spectrum of the periodic orbit under modulation shown in Fig. 6(c). The inset shows the details of the modulated fundamental harmonic. Because the frequency of the $n$th harmonic is $n$ times larger than that of the fundamental, the frequency deviation of the $n$th harmonic is $n$ times larger than that of the fundamental subcarrier. The FM modulation index and, potentially, FM demodulation gain increase with the order of the subcarrier. The existence of the harmonics increases the frequency of the subcarrier and peak frequency deviation for a given signal power, and as such offers both flexibility and restrictions in system and network applications. The effect of a larger peak frequency deviation for a given signal power shifts the dynamic range of the system toward lower signal powers. This is because a specified signal-to-noise ratio/modulation index can be achieved with lower signal powers with high-order harmonics in the periodic orbits. The dynamic range of a system using a highorder harmonic, however, may not necessarily increase. This is because the large peak frequency deviation of a high-order harmonic at relatively low modulating powers may cause its sidebands to overlap with sidebands of its neighboring lower-order harmonic such as shown for the second and third harmonics in Fig. 6(c).

\section{SYSTEM PERFORMANCE ESTIMATES}

Next, we present the performance of the subcarrier optical communication systems based on the optical CCO. The schematic shown in Fig. 7 represents one of the possible ways of utilizing the optical CCO for subcarrier optical communications. On the transmitter side, even though microwave subcarriers are generated, no microwave components are used. The dc bias to the CCO establishes the desired subcarrier frequency. The information is superimposed on the dc bias via a bias tee. The output of the CCO is coupled into a fiber and delivered to the receiving end. What is trans- mitted in the fiber is an amplitude/frequency-modulated periodic-orbit subcarrier on an optical carrier. When the optical carrier is stripped off with photodetection, the resultant electric signal is an amplitude/frequency-modulated periodic-orbit signal. If we select only one harmonic by band pass filtering, the signal will be an AM/FM modulated sinusoidal carrier. The limiter is used to eliminate residual amplitude modulation so that, after the limiter, the signal is a conventional FM signal, which can be demodulated by conventional FM demodulation techniques such as the delay-line type of demodulator shown here. We choose to use the FM format because of the potential FM demodulation gain. ${ }^{31}$ The numerical simulation is based on the two-section FP CCO and the delay-line demodulator. Because of the particular dynamics of the two-section FP CCO and the delay-line demodulator's ability to discriminate against AM, the use of the limiter does not offer significant benefits to system performance. So the results presented here correspond to no amplitude limiting. Stochastic noises are not included in the present evaluation of system performance. Instead, we focus on systematic nonlinear distortions due to the deterministic characteristics of the optical CCOs. Specifically, we simulate the system performance under the scenario that the message contains two harmonic signals at frequencies $f_{1}$ and $f_{2}$ with equal power. In addition to recovering the two harmonic signals at frequencies $f_{1}$ and $f_{2}$ after the demodulator, secondorder intermodulation distortion at frequencies $f_{1}$ $\pm f_{2}, 2 f_{1}, 2 f_{2}$, and third-order distortion intermodulation at frequencies $2 f_{1} \pm f_{2}, 2 f_{2} \pm f_{1}, 3 f_{1}, 3 f_{2}$ are also detected. These distortions are accumulated as a result of the nonlinear dependence of the subcarrier frequency as well as the residual amplitude and pulse width modulation.

\section{A. Intermodulation distortion as a function of modulating power}

Figure 8 shows the power contained in the second- (at $f_{1}+f_{2}$ ) and third-order (at $\left.2 f_{1}-f_{2}\right)$ intermodulation distortions (IMDs), normalized by the received signal power (at $f_{1}$ ), as functions of the power in harmonic signal at $f_{1}$ in the message at the transmitter using the fundamental and the second harmonic of the periodic orbits as the subcarrier (fundamental and second-order subcarriers). As expected the slope ratio of the normalized second-order IMD to the thirdorder IMD is 1:2 (2:3 un-normalized). The second-order IMD is practically the same when the subcarrier is the fundamental or the second harmonic of the periodic orbit. For the third-order IMD, however, the second-harmonic subcarrier offers a $10 \mathrm{~dB}$ improvement over the fundamental subcarrier. The second-order IMD is below $20 \mathrm{~dB}$ for modulating powers below $-8 \mathrm{~dB} \mathrm{~m}$. The third-order IMD is below $55 \mathrm{~dB}$ for modulating powers below $-11 \mathrm{~dB} \mathrm{~m}$ for the fundamental subcarrier and $-7 \mathrm{~dB} \mathrm{~m}$ for the second-harmonic subcarrier. The second-order IMD can be avoided by limiting the information bandwidth to within one octave and using appropriate band pass filtering. It turns out that, for this $\mathrm{CCO}$, the second-harmonic subcarrier offers a $10 \mathrm{~dB}$ improvement in the third-order IMD over the fundamental subcarrier. 


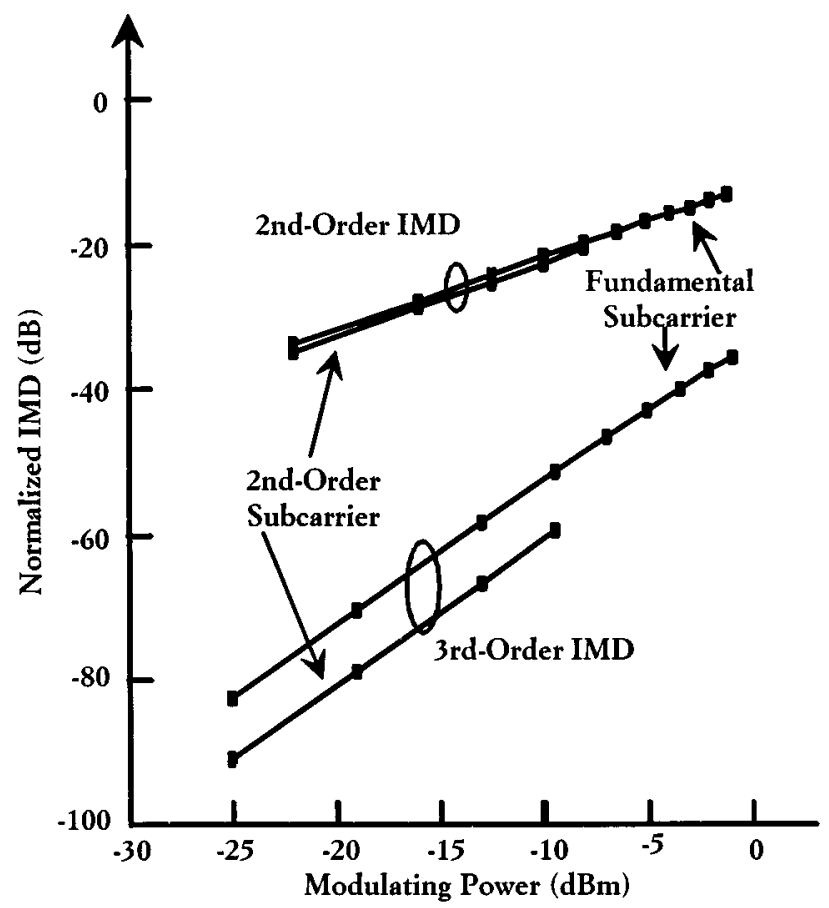

FIG. 8. Normalized IMDs using fundamental and second-order subcarriers as functions of the power of the modulating signal.

\section{B. Dependence of the power budget and intermodulation distortion on the order of the subcarrier}

We next investigate the performance of the system using different harmonic subcarriers. We assume that the bandwidth of each subcarrier channel is limited (to $180 \mathrm{MHz}$, arbitrarily chosen here) as is normally the case in network applications. Under this assumption, the modulating power decreases with the order of the subcarrier so that the modulated subcarriers occupy the same amount of bandwidth. The unmodulated subcarrier power determines the maximum power loss allowed from the transmitter to the receiver. In view of this, we also include the unmodulated subcarrier power, in $\mathrm{dB}$ relative to that of the fundamental harmonic, together with the second- and third-order IMDs for different subcarrier orders in Fig. 9. The fundamental subcarrier frequency is $1.5 \mathrm{GHz}$, and the modulating signal power is adjusted for the specified bandwidth. It is observed that the power budget in the second-order subcarrier is only about 1 $\mathrm{dB}$ below the fundamental subcarrier. But the power budget in the third-order subcarrier is $3 \mathrm{~dB}$ below and it decreases progressively as the order of the subcarrier is increased. Second-order IMD decreases as the order of the subcarrier increases. The third-order IMD decreases for the secondorder and third-order subcarriers, and then saturates for higher orders. These dependences are the consequences of the complicated nonlinear dynamics of the two-section FP LD. When other CCO architectures are employed, these dependences will likely change and need to be explored so as to select the optimum order of subcarrier from the periodic orbits.

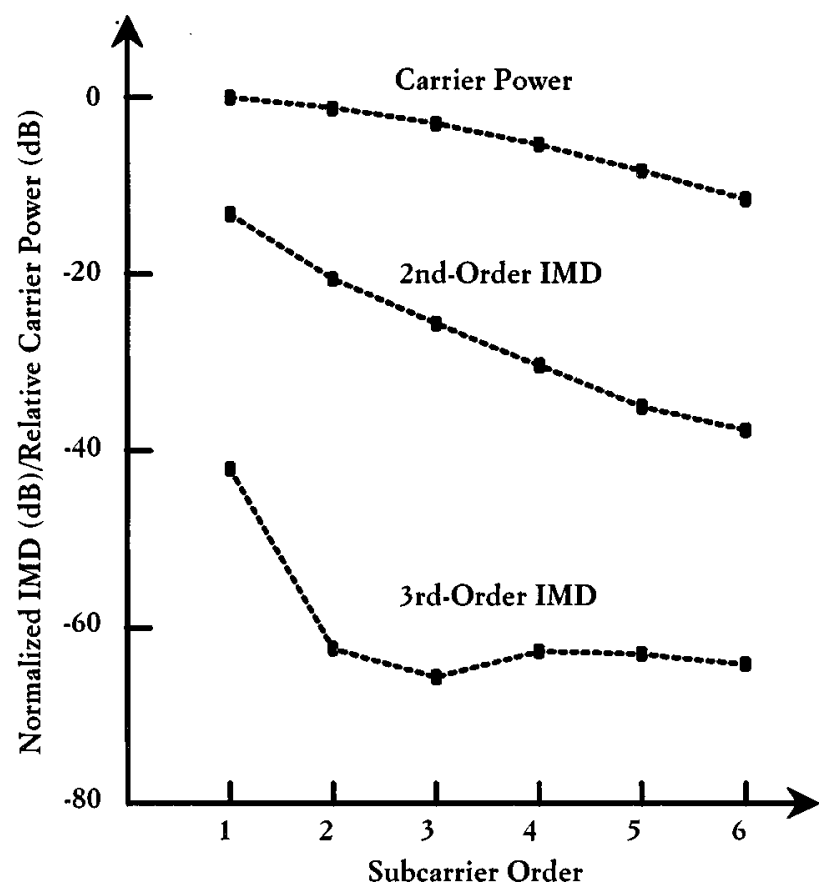

FIG. 9. Dependence of the normalized IMDs and the power of the unmodulated subcarrier, in $\mathrm{dB}$ relative to that of the fundamental, on the subcarrier order.

\section{Choice of dc bias}

Since the characteristics of the periodic orbits depend on the dc bias level, the performance of the system is strongly dependent upon the dc bias also. These dependencies are illustrated in Fig. 10. The top traces are the fundamental and second-order subcarrier powers as functions of the injection current, shown here for comparison. For each dc bias current, the maximum frequency deviation is again limited to 180 MHz. Both the second-order and the third-order IMDs exhibit minimum values for particular injection currents. For the same order of the subcarrier, the optimum injection currents are different for minimum second-order and third-order IMDs. Further, the optimum injection currents for minimum second (third)-order IMD are different for different orders of the subcarriers. The optimum injection current for minimum second-order IMD matches the injection current at which the subcarrier power is peaked [see Fig. 5(c)] where the residual amplitude modulation is minimum. It is easily shown that residual amplitude modulations lead to second-order IMD. ${ }^{32}$ The $10 \mathrm{~dB}$ antiresonance thus roughly represents the contribution from residual AM, which can be eliminated by the use of a limiter. Since it is not possible to reduce the secondorder IMD to below $55 \mathrm{~dB}$ with amplitude limiting and the third-order IMD is below $55 \mathrm{~dB}$ in the absence of amplitude limiting, the use of a limiter will not relax the requirement of $\mathrm{CCO}$ for a specified network capacity (see Sec. VII). Thus, the limiter is not used for the current simulation.

\section{ARCHITECTURES AND CAPACITY OF A CCO- BASED SCMA NETWORK}

Optical CCO-based SCMA networks represent one of the practical applications of CCO technology. Such a net- 


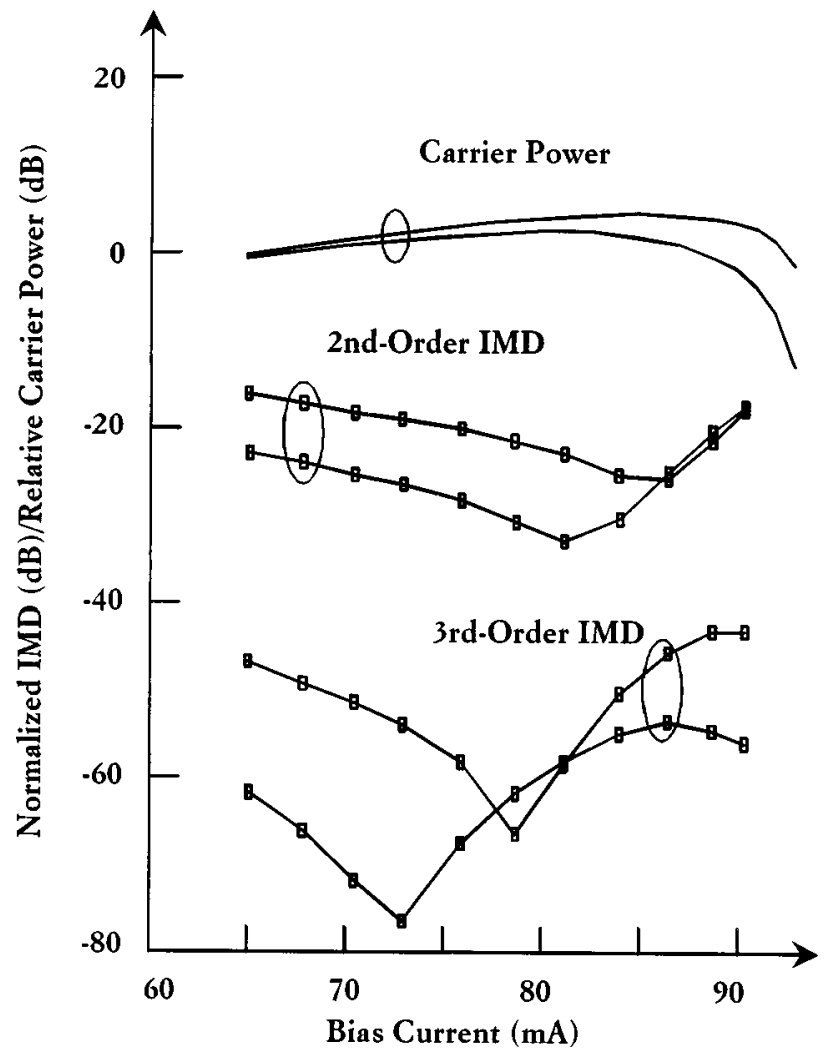

FIG. 10. Normalized IMDs and the power of the unmodulated subcarrier, in $\mathrm{dB}$ relative to that of the fundamental at $65 \mathrm{~mA}$, as functions of the bias current. In each case, the upper trace is for the fundamental subcarrier and the lower trace is for the second-order subcarrier.

work may be a suitable candidate for multiaccess local networks. The CCO-based SCMA networks do not rely on an electronic component to generate subcarriers. This is the fundamental difference between CCO-based and conventional SCMA networks. The broadcast-and-select network based on star couplers and CCOs as tunable transmitters and/or tunable local oscillators (LOs) in receivers, shown in Fig. 11, is the simplest multiaccess architecture. In such SCMA networks, CCOs replace the role traditionally played by VCOs at the transmitter and receiver ends. Multiaccess is achieved by using tunable CCOs in the transmitter and fixedfrequency LOs in the transmitter or by assigning a fixed $\mathrm{CCO}$ subcarrier frequency at the transmitter and using tunable CCOs or VCOs at the receiver end (compare Node \#N with Node \#1).

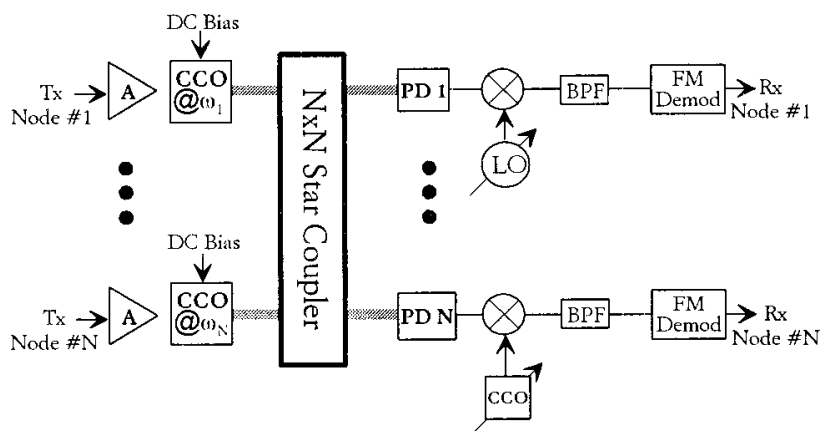

FIG. 11. Schematic of a CCO-based subcarrier multiaccess optical network.
The capacity of the SCM network is limited by the fundamental frequency of the periodic-orbit subcarrier. Let the single-channel baseband information of bandwidth $W$ occupy frequencies from $f_{l}$ to $f_{h}, N$ be the number of channels (nodes) in the network, $f_{0}$ be the fundamental frequency of the periodic-orbit subcarrier, and $\beta$ be the modulation index. It can be shown in a straightforward manner that the information capacity $N \times W$ is independent of the order of the subcarrier used. Subcarrier multiplexing/multiple access requires that

$$
f_{0} \geqslant[(4 N-1) \beta+4 N-2]\left(f_{h}-f_{l}\right),
$$

where $f_{l}=W, f_{h}=2 W$ for analog networks and $f_{l}=0$, $f_{h}=W$ for digital networks. The reason for the increased bandwidth requirement for analog transmission is because the modulating signal for each CCO should occupy only one octave or less in the frequency domain to avoid second-order IMD, discussed in Sec. VI. Assuming $\beta=1$ and using Eq. (5), one can estimate that, in order to transmit 100 digital channels with $52.84 \mathrm{MHz} /$ channel, the minimum CCO frequency required is $41.472 \mathrm{GHz}$.

\section{CONCLUSION AND DISCUSSIONS}

We have presented the theoretical foundation of using periodic orbits in nonlinear optical systems as subcarriers for all-optical subcarrier systems and networks. The optical $\mathrm{CCO}$ is a new technology that exploits instabilities in nonlinear systems. Optical CCO-based subcarrier systems and networks represent a paradigmatic shift from the conventional subcarrier systems in which electronic components are used for subcarrier generation. Since optical CCO technology is relatively new, much work remains to be done.

It is important to outline the differences between CCOs and pulsed lasers (in particular mode-locked lasers). First, traditional mode-locking mechanisms such as saturable absorption are not required for the CCOs. Second, the repetition rates/frequency of periodic orbits in CCOs are broadly tunable while in mode-locked lasers the pulse repetition rates are fixed and equal to the cavity mode spacing. Third, the ideal periodic orbits in CCOs are, in general, sinusoidal so as to be used as subcarriers while for mode-locked lasers the goal is to achieve ultrashort pulse width.

The frequency of optical CCOs based on two-section FP LDs will be limited by the carrier lifetimes. Other CCO architecture can be identified and engineered since the formation of periodic orbits is a ubiquitous phenomenon in nonlinear systems. The following are two examples that are worth further consideration and investigation. First, a single-stripe weakly index-guided semiconductor LD exhibits highfrequency (100 GHz range) tunable periodic-orbit formation due to lateral mode instability. ${ }^{33}$ Another example is the twosection distributed feedback laser, which has resulted in tunable periodic orbits up to $80 \mathrm{GHz}{ }^{34}$

We have not included the effects of stochastic noises in the present system performance simulation. To include such an effect would be difficult because there is no known methodology for predicting the noise characteristics of periodic orbits. The noise performance of the $\mathrm{CCO}$, the modulation 
bandwidth, as well as other modulation format and associated demodulation techniques are currently under investigation.

\section{ACKNOWLEDGMENTS}

This research was supported in part by the NSF CAREER program under Contract No. ECS-9622298, the ONR Young Investigator program under Contract No. N00014-95-1-0966, U.S. Air Force Rome Laboratory under Contract No. F30603-95-C-0091, and the Gleason Memorial Fund of Rochester, NY.

${ }^{1}$ For a review, see IEEE J. Sel. Areas Commun. 8 special issue on applications of rf and microwave subcarriers to optical fiber transmission in present and future broadband networks; see also IEEE Lightwave Commun. Syst. 1, special issue on optical fiber video delivery systems of the future.

${ }^{2}$ T. E. Darcie, J. Lightwave Technol. LT-5, 1103 (1987).

${ }^{3}$ W. I. Way, R. S. Wolff, and M. Krain, J. Lightwave Technol. LT-5, 1325 (1987).

${ }^{4}$ T. E. Darcie et al., Electron. Lett. 22, 774 (1986).

${ }^{5}$ T. E. Darcie et al., J. Lightwave Technol. LT-5, 1103 (1989).

${ }^{6}$ M. M. Choy, S. M. Altieri, and K. N. Sivarajan, Proc. SPIE 1787, 32 (1992).

${ }^{7}$ P. J. Heim and C. P. McClay, IEEE Trans. Microwave Theory Tech. 38, 494 (1990)

${ }^{8}$ A. Budman, E. Eichen, J. Schlafer, R. Olshansky, and F. McAleavey, Proceedings of the Optical Fiber Communication Conference, San Jose, CA, February 23-28, 1992, pp. 90-91.

${ }^{9}$ M. D. Vaugh, A. Wang, and D. J. Blumenthal, Proceedings of the Optical Fiber Communication Conference, San Diego, CA, February 26-March 3, 1995, pp. 128-129.

${ }^{10}$ E. Park and A. E. Wilner, in Ref. 9, pp. 114-115.

${ }^{11}$ R. Olshansky et al., J. Lightwave Technol. LT-7, 1329 (1989).

${ }^{12}$ W. I. Way, J. Lightwave Technol. LT-7, 1806 (1989).

${ }^{13}$ P. M. Hill et al., J. Lightwave Technol. 8, 554 (1990).
${ }^{14}$ T. E. Darcie et al., J. Lightwave Technol. LT-7, 997 (1989).

${ }^{15}$ X. Wang and G. Li, Proc. SPIE 1703, 163 (1992).

${ }^{16}$ X. Wang, G. Li, and C. S. Ih, J. Lightwave Technol. 11, 309 (1993).

${ }^{17}$ G. Li, Proc. SPIE 2481, 62 (1995).

${ }^{18}$ G. Li, R. K. Boncek, X. Wang, and D. H. Sackett, IEEE Photonics Technol. Lett. 7, 854 (1995).

${ }^{19}$ J. B. Georges and K. Y. Lau, IEEE Photonics Technol. Lett. 4, 662 (1992).

${ }^{20}$ J. B. Georges and K. Y. Lau, IEEE Photonics Technol. Lett. 5, 242 (1993).

${ }^{21}$ J. Guckenheimer and P. Holmes, Nonlinear Oscillations, Dynamical Systems and Bifurcations of Vector Fields (Springer, New York, 1983).

${ }^{22}$ J. M. T. Thompson and H. B. Stewart, Nonlinear Dynamics and Chaos: Geometrical Methods for Engineers and Scientists (Wiley, New York, 1986).

${ }^{23}$ T. L. Paoli and J. Ripper, Appl. Phys. Lett. 15, 105 (1969).

${ }^{24}$ J. P. van der Ziel, J. L. Merz, and T. L. Paoli, J. Appl. Phys. 50, 4620 (1979).

${ }^{25}$ K. D. Chik, J. C. Dyment, and B. A. Richardson, J. Appl. Phys. 51, 4029 (1980).

${ }^{26}$ M. Ueno and R. Lang, J. Appl. Phys. 58, 1689 (1985).

${ }^{27}$ R. W. Dixon and W. B. Joyce, IEEE J. Quantum Electron. QE-15, 470 (1979).

${ }^{28}$ K. Y. Lau and A. Yariv, Appl. Phys. Lett. 45, 124 (1984).

${ }^{29}$ T. H. Wood and N. K. Shankaranarayanan, J. Lightwave Technol. 11, 1632 (1993). In this work, the modulation index was measured to be above $100 \%$. It is unclear from the data whether clipping has occurred and whether analog applications are possible since only digital data was used in the experiment.

${ }^{30}$ D. J. G. Mestdagh, Fundamentals of Multiaccess Optical Fiber Networks (Artech House, Dedham, MA, 1995), p. 315.

${ }^{31}$ L. W. Couch II, Modern Communication Systems-Principles and Applications (Prentice-Hall, Englewood Cliffs, NJ, 1995), p. 337.

${ }^{32}$ F. G. Stremler, Introduction to Communication Systems 3rd ed. (AddisonWesley, Reading, MA, 1990), p. 305.

${ }^{33}$ L. Rahman and H. G. Winful, IEEE J. Quantum Electron. 30, 1405 (1994).

${ }^{34}$ U. Feiste, D. J. As, and A. Erhardt, IEEE Photonics Technol. Lett. 6, 106 (1994). 